\title{
User Association in 5G Heterogeneous Networks with Mesh Millimeter Wave Backhaul Links
}

\author{
Agapi Mesodiakaki, Enrica Zola ${ }^{\dagger}$, and Andreas Kassler ${ }^{\star}$ \\ ${ }^{\star}$ Department of Computer Science, Karlstad University, Karlstad, Sweden \\ ${ }^{\dagger}$ Dept. of Network Engineering, Universitat Politècnica de Catalunya, Barcelona, Spain \\ Email: \{agapi.mesodiakaki, andreas.kassler\}@kau.se, enrica@entel.upc.edu
}

\begin{abstract}
Fifth generation (5G) wireless networks will target at energy and spectrum efficient solutions to cope with the increasing demands in capacity and energy efficiency. To achieve this joint goal, dense networks of small cells (SCs) are expected to overlay the existing macro cells. In parallel, for the $\mathrm{SC}$ connection to the core network, a promising solution lies in a mesh network of high capacity millimeter wave backhaul (BH) links. In the considered 5G architecture, each SC is able to forward its BH traffic to the core network through alternative paths, thus offering high $\mathrm{BH}$ network reliability. In this context, the joint problem of user association and $\mathrm{BH}$ routing becomes challenging. In this paper, we focus on this problem targeting at energy and spectrum efficient solutions. A low-complexity algorithm is proposed, which bases its user association and $\mathrm{BH}$ routing decision i) on minimizing the spectrum resources to guarantee the user rate, so as to provide high spectrum efficiency, and ii) on minimizing both the access network and $\mathrm{BH}$ route power consumption to provide high energy efficiency. Our results show that our solution provides better trade-offs between energy and spectrum efficiency than the state-of-the-art in 3GPP scenarios.
\end{abstract}

Index Terms-5G, backhaul, cell selection, context-awareness, green networks, LTE-Advanced, mesh, millimeter wave, routing.

\section{INTRODUCTION}

A NNUAL global IP traffic will reach 2.3 ZB by 2020 (ZB; 1000 exabytes [EB]), while there will be three times more connected devices than our global population in 2020 [1]. To deal with these demands, energy and spectrum efficient wireless solutions, able to offer high capacity, are needed.

As a result, the nature of fifth generation (5G) wireless networks is expected to be heterogeneous, consisting of a dense network of small cells (SCs) deployed on the top of the existing macro cells [2]. The benefits of the dense SC deployment are threefold. Firstly, the user comes closer to its serving base station (BS), which results in higher signal-tointerference-plus-noise ratio (SINR), and thus, higher capacity as well as lower mobile battery consumption. Secondly, frequency reuse can be applied among SCs that are located far from each other, hence offering higher area spectrum efficiency. Thirdly, millimeter wave (mmWave) is favored to offer high capacity wireless backhaul (BH) links, i.e., set of links between the BSs and the core network. This is mainly due to two reasons: 1) the connection of each SC to the core by fiber is highly cost-inefficient and 2) the anticipated short $\mathrm{BH}$ link length among neighboring SCs will result in line-of-sight (LOS) opportunities, essential for good mmWave coverage.

In particular, most macro cells are already connected through fiber to the core. Therefore, exploiting the existing connection and providing core connectivity to SCs through it with the use of mmWave links seems promising. As a result, a mesh BH network of LOS mmWave links is expected, where each SC will forward its traffic to its neighbors, selecting among a broad set of alternative paths, to reach the core. This topology combines the mmWave benefits with the mesh networking advantages. On the one hand, mmWave offers high spectrum availability, and consequently, high capacity links. In addition, the very small mmWave wavelength enables higher antenna gains, resulting in highly directional links. Therefore, mmWave is able to compensate the higher path loss experienced at higher frequencies [3]. On the other hand, mesh networking can increase reliability and redundancies through self-forming and self-healing in case of a BH link failure [2].

In this context of hyper-dense $5 \mathrm{G}$ heterogeneous networks with complicated $\mathrm{BH}$ topologies, selecting the serving $\mathrm{BS}$ of a user equipment (UE), becomes challenging, as it impacts both the network and UE performance. Hence, new low-complexity UE association and BH traffic routing algorithms are needed, able to maximize the network energy and spectrum efficiency.

However, the majority of user association algorithms proposed so far focus on the performance optimization of the access network (AN), i.e., the links between the UEs and their serving BSs. Specifically, LTE-Advanced (LTE-A) employs two metrics: the reference signal received power (RSRP) and the reference signal received quality (RSRQ) [4]. Equivalently, the best-SINR algorithm connects a UE to the BS with the highest received power. Although the aforementioned criteria maximize the spectrum efficiency, they do not maximize the network throughput, as few UEs connect to SCs. This limitation was overcome by range expansion (RE), where a bias was applied for signals originated by SCs [5]. Thereby, the connections with SCs were favored, resulting in load balancing between SCs and macro. Finally, in the extreme biasing case, a UE connects to the BS with the lowest experienced path loss, i.e., minimum path loss (MPL) [6]. MPL achieves the highest offloading to SCs at the expense of low spectrum efficiency.

On the other hand, there are few works that consider the $\mathrm{BH}$ conditions in the user association decision. In particular, [7] proposes a user association analytical framework, which jointly considers the AN and BH. Specifically, spectrum efficiency, base station load, BH link capacity and topology, as well as different types of traffic are taken into account. In [8], the authors study the joint problem of user association and resource allocation, considering the resource consumption 
and the energy budget of BSs, as well as the maximum $\mathrm{BH}$ capacity. However, in all these BH-aware approaches, there is no study of energy consumption, and hence, their high performance in terms of energy efficiency cannot be ensured.

To this end, in [9], [10], the authors study the aforementioned problem focusing on the energy and spectrum efficiency maximization of a network with tree BH links. Specifically, the algorithm proposed in [9] selects among the BSs that maximize the network spectrum efficiency, while taking into account the number of BH link hops to reach the core network. Nevertheless, due to the simplicity of the applied criterion, its high energy efficiency in scenarios with heterogeneous $\mathrm{BH}$ links, i.e., links that differ in length, allocated bandwidth, or even in applied frequency, cannot be ensured. Therefore, in [10], the authors proposed a solution that takes into account the amount of power consumed in each BH link, thereby relaxing the limitation of homogeneous BH links. Still, in both works, the proposed solutions focus on tree topologies, where a single BH link route is available for each SC. However, in such 5G networks consisting of mesh $\mathrm{BH}$ links, the $\mathrm{BH}$ routing problem is another challenge that should be jointly considered.

Therefore, in this paper, we study the joint problem of user association and $\mathrm{BH}$ traffic routing with the aim of maximizing the energy and spectrum efficiency of the network, while guaranteeing the UE quality of service (QoS). A low-complexity UE association algorithm, which is able to provide good tradeoffs between the two competitive objectives. Moreover, in order to demonstrate the benefits of load balancing in the algorithm performance, two different cases are studied, i.e., with or without load balancing. Finally, the proposed algorithm is compared with existing solutions and we demonstrate notable performance gains for our approach.

The rest of the paper is organized as follows. In Section II, the system model is presented. Sections III and IV include the description of our proposal and its comparison with existing solutions in 3GPP-compatible scenarios, respectively. Finally, Section V concludes the paper.

\section{SySTEM MOdEL}

According to 3GPP [11], we consider an LTE-A grid of three-sectorized eNodeBs (eNBs), as shown in Fig. 1. Each eNB sector is overlaid with SCs, which are grouped into clusters. The set of BSs (eNBs and SCs) is defined by $\mathcal{B}$. We focus on the downlink case and we assume the following.

- There is a fiber connection between each eNB and the core. The SCs exploit this connection, and thus, access the core network indirectly through the eNB site [2], [4].

- For the connection of SCs to the eNB site, a mesh network of $L \operatorname{LOS}^{1} \mathrm{BH}$ links, operating at mmWave frequencies, is considered, denoted by the set $\mathcal{L}$.

- We consider flat slow-fading AN channels [6]. Therefore, we assume equal distribution of the total transmission power of each BS $j\left(P_{j_{\max }}\right)$ to its subcarriers [4]. Moreover, each $\mathrm{BS} j$ is provided with a maximum number of physical resource blocks (PRBs), denoted by $c_{j_{\max }}$. As

\footnotetext{
${ }^{1}$ Our study could also be applied to non-LOS BH links. However, in this case, the performance of mmWave deteriorates significantly. Thus, to fully exploit the mmWave benefits, we consider a number of hops to achieve LOS.
}

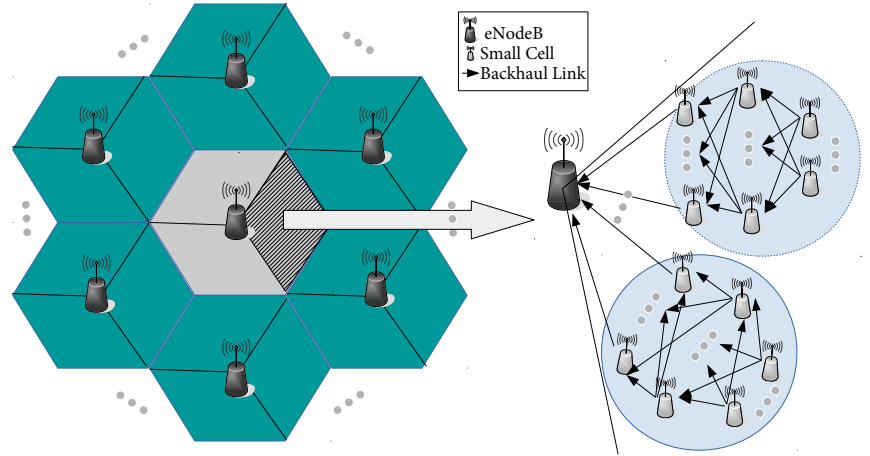

Fig. 1. System model.

a result, the power allocated per PRB by each BS $j$ is constant and equal to $P_{j_{P R B}(W)}=P_{j_{\max (W)} / c_{j_{\max }}}$

- We consider a set $\mathcal{U}$ of UEs with strict guaranteed bit rate (GBR) QoS requirements [12], defined as $r_{u}$.

- The UE association with a single BS at a time is allowed.

- The link vector $x_{(i, j)}^{u}$ defines every AN or BH link in the network that carries the traffic of user $u \in \mathcal{U}$ between two nodes $i$ and $j$. It is equal to 1 when the traffic of user $u$ passes through the link $(i, j)$ and 0 otherwise. Note that $i \in \mathcal{B}$ and $j \in \mathcal{U}$ in the case of an AN link, whereas $i, j \in \mathcal{B}$ in the case of a BH link. In the first case, the link vector $x_{(i, u)}^{u}$ can be interchangeably used, whereas in the latter, the $x_{l}^{u}$, where link $(i, j)$ is equivalent to BH link $l$.

To calculate the total energy efficiency of the network, the $\mathrm{AN}$ and $\mathrm{BH}$ models for power consumption are provided in the following. In particular, the total power of the network consists of the $\mathrm{AN}$ and the $\mathrm{BH}$ power consumption, consumed in the BSs and in the BH links, respectively. The first equals to [13]

$$
P_{A N_{(W)}}=\sum_{i \in \mathcal{B}} N_{T R X_{i}}\left(P_{0_{i(W)}}+\Delta_{p_{i}} P_{\text {out }_{i(W)}}\right),
$$

where $N_{T R X_{i}}$ is the number of transceiver chains of BS $i$, $P_{0_{i}}$ is the static power consumption of BS $i$ related to, e.g., cooling, power supply and baseband unit operation [13] and $P_{\text {out }_{i}}$ is its load-dependent transmit power consumption. To account for the non-ideal power efficiency of the electronics, we also define with $\Delta_{p_{i}}$ the slope indicating how the transmitted power of BS $i$ scales with the traffic. The transmit power consumption part of BS $i \in \mathcal{B}$ is then given as [9]

$$
P_{\text {out }_{i(W)}}=\sum_{u \in \mathcal{U}} P_{i_{P R B}(W)}[\overbrace{\frac{r_{u}}{B W_{P R B} S E_{(i, u)}}}^{c_{i u}}] x_{(i, u)}^{u},
$$

where $c_{i u}$ stands for the number of PRBs required for the association of UE $u$ with BS $i$ and $r_{u}$ is the demanded rate of UE $u . B W_{P R B}$ is the bandwidth of a PRB and $\lceil\cdot\rceil$ is the ceiling function operator. Then, the maximum achievable spectrum efficiency with effective $S I N R_{(i, u)}$ [14] is given by

$S E_{(i, u)}=\min \left(N_{T X_{i}}, N_{R X_{u}}\right) \log _{2}\left(1+\frac{N_{R X_{u}} S I N R_{(i, u)}}{\min \left(N_{T X_{i}}, N_{R X_{u}}\right)}\right)$,

with $N_{T X_{i}}, N_{R X_{u}}$ representing the number of transmitting and receiving antennas of BS $i$ and UE $u$, respectively. Finally, $x_{(i, u)}^{u}$ is the link vector (in this AN link case, it is equal to 1 , when UE $u$ is associated with BS $i$, and 0 otherwise). 
As in the $\mathrm{AN}$, the $\mathrm{BH}$ power consumption ${ }^{2}$ includes a fixed and a variable part, and hence, can be given by [13]

$$
P_{B H_{(W)}}=\sum_{l \in \mathcal{L}} N_{T R X_{l}}\left(P_{0_{l}(W)}+\Delta_{p_{l}} P_{\text {out }_{l}(W)}\right) .
$$

The transmit power consumption part of a $\mathrm{BH}$ link $l$ operating in the mmWave band is given by [3]

$$
\overbrace{+L_{\text {out }_{l(d B m)}}=S M_{(d B)}+N_{T H(d B m)}+N R_{l}^{\text {trg }}(d B)}+\overbrace{L_{T X_{l(d B)}}+L_{R X_{l(d B)}}+P L_{l(d B)}}^{\beta_{l(d B m)}},
$$

where $L_{T X_{l}}, L_{R X_{l}}$ and $G_{T X_{l}}, G_{R X_{l}}$ are the losses and the antenna gains, respectively, of the transmitter-receiver pair of BH link $l$. The parameter $P L_{l}$ stands for the path loss of the link (sum of free space path loss, rain and gas attenuation [3]), $L M$ is the link margin, $N_{T H}$ is the thermal noise, and $N F_{B H}$ is the noise figure of the receiver. From now on, we denote with $\beta_{l}$ the overbraced equation, which results after the subtraction from the total losses of the BH link $l$ the gains of its transmitter-receiver pair. Finally, we employ link adaptation [14], and hence, $S I N R_{l}^{t r g}$ is the target SINR for the traffic of BH link $l$ to be successfully delivered, which equals to [14]

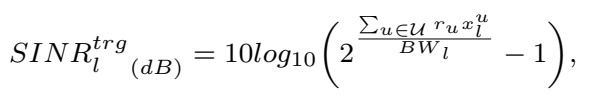

where $B W_{l}$ is the bandwidth of the BH link $l$ and $\sum_{u \in \mathcal{U}} r_{u} x_{l}^{u}$ is the aggregate traffic that passes through it. The generated interference of mmWave is negligible due to high path loss and highly directional antennas, and thus, $S I N R_{l}^{t r g}=S N R_{l}^{t r g}$.

It is worth noting that in the following sections, we focus on the variable power consumption part of the aforementioned power models, given that a different user association decision would only impact this load-dependent part.

\section{Proposed energy and SPeCtrum efFicient User ASSOCIATION AND BACKHAUL ROUTING ALGORITHM}

In this section, we propose a low-complexity algorithm for joint user association and $\mathrm{BH}$ traffic routing, which targets at a good trade-off between energy and spectrum efficiency. From here on, it will referred to as energy and spectrum efficient (ESE) algorithm. ESE considers: 1) the UEs' measurements and requirements, 2) the $\mathrm{AN}$ and $\mathrm{BH}$ topology, and 3) the available capacity of each BS $i$ (in terms of maximum spectrum resources) and BH link $l$ (in terms of maximum transmission power for given bandwidth) to efficiently associate the UEs, taking into account the power and resource consumption in both the AN and BH links. In particular, ESE targets at maximizing the energy efficiency, while satisfying a spectrum efficiency constraint, controlled by parameter $\delta_{\text {thres }}$. Given that that each UE GBR demand should be satisfied, the total energy efficiency maximization is equivalent to the total power consumption minimization, i.e., power consumed in the BSs

\footnotetext{
${ }^{2}$ In this study, we focus on power and throughput optimization. We acknowledge that packet latency will be affected by our decision making process, as some flows are routed over multi-hop paths while others are sent directly to the eNB from the UEs. However, latency is a much more complex parameter to optimize due to packet scheduling policies and arrival rate at the intermediate nodes, which is out of the scope of this paper.
}

and in the mesh BH links, while the spectrum efficiency is only related to the number of required PRBs.

To provide high spectrum efficiency, ESE selects as best associations for a UE $u$ the subset of BSs that guarantee its rate demand with fewer PRBs than a target, i.e., $c_{i u} \leq$ $\min \left(\delta_{\text {thres }} c_{i u_{\min }}, c_{i_{\max }}\right)$, where $c_{i u_{\text {min }}}$ refers to the association with the cell that requires the fewest PRBs to guarantee $r_{u}$. For instance, for a UE demanding 3 PRBs from eNB, 4 PRBs from SC 1, 5 PRBs from SC 2 and 3 PRBs from SC 3 to satisfy its QoS, $c_{i u_{\min }}=3$. As already explained, the spectrum efficiency target is controlled by $\delta_{\text {thres }} \geq 1$, which reflects the deviation in terms of PRBs between the selected association and the one that requires the fewest. Hence, $\delta_{\text {thres }}=1$ leads to maximum spectrum efficiency (in the previous example, the eNB and SC 3 would be selected for possible association), whereas $\delta_{\text {thres }}>1$ decreases spectrum efficiency in favor of higher energy efficiency (e.g., with $\delta_{\text {thres }}=1.5, c_{i u_{m i n}} \leq$ $1.5 \times 3=4.5$, and thus possible candidates would be the eNB and SCs 1 and 3). Moreover, a BS $i$ is not considered for possible association with a UE $u$, if $S I N R_{(i, u)}$ is too low, and hence, $c_{i u}>c_{i_{\max }}$. To guarantee that all UEs get associated, ESE decides first the association of UEs with few candidates.

To maximize energy efficiency, ESE calculates for each UE $u$ and candidate BS $i$ the total power consumption, i.e., sum of the AN $\left(P_{A N_{(i, u)}}\right)$ and $\mathrm{BH}$ power consumption $\left(P_{B H_{u}^{t}}\right)$, for its traffic to be served, denoted by $P_{t o t}^{t}{ }_{(i, u)}$. From (1)-(3) and only focusing on the variable part, $P_{A N_{(i, u)}}$ is equal to

$$
P_{A N_{(i, u)}(W)}=N_{T R X_{i}} \Delta_{p_{i}} P_{i_{P R B(W)}} c_{i u} .
$$

As for the BH power consumption $P_{B H_{u}^{t}}$, when UE $u$ gets associated with candidate $\mathrm{BS} i$, there may be more than one alternative $\mathrm{BH}$ routes. Hence, for every different $\mathrm{BH}$ route $t$, from (4)-(5) and only focusing on the variable part, we have

$$
P_{B H_{u}^{t}(W)}=\sum_{l \in \mathcal{L}_{t}^{u}} N_{T R X_{l}} \Delta_{p_{l}} \operatorname{SINR}_{l}^{\operatorname{trg}}(W) \beta_{l(W)},
$$

where $\mathcal{L}_{t}^{u} \subseteq \mathcal{L}$ refers to the set of links that belong to the $\mathrm{BH}$ route $t$, which is followed by the traffic of UE $u$ until it reaches the eNB site. Given the mesh BH topology, two different cases are considered: with and without load balancing, which differ on how the parameter $S I N R_{l}^{\operatorname{trg}}$ is estimated in (8).

- ESE without load balancing: In the calculation of $P_{B H_{u}^{t}}$, ESE takes into account the BH power consumption of the links $l$ that belong to the route $t$ as if they were exclusively used by UE $u$. In other words, for the calculation of $S I N R_{l}^{t r g}$, the following equation is used

$$
\operatorname{SINR}_{l}^{\operatorname{trg}}(W)=2^{\frac{r_{u}}{B W_{l}}}-1 .
$$

- ESE with load balancing: In the calculation of $P_{B H_{u}^{t}}$, ESE takes into account the BH power consumption of the BH links $l$ that belong to the route $t$ by considering both the traffic of the UE $u$ and the already associated UEs (whose traffic is also served through the considered BH link). In other words, if we denote with $\mathcal{U}_{\text {assoc }} \subseteq \mathcal{U}$, the set of UEs that are already associated, the parameter $S I N R_{l}^{t r g}$ is calculated, in this case, as follows

$$
\operatorname{SINR}_{l}^{\operatorname{trg}}(W)=2^{\frac{\left(\sum_{i \in \mathcal{U}_{a s s o c}} r_{i} x_{l}^{i}\right)+r_{u}}{B W_{l}}}-1 .
$$


Thereafter, in both approaches, to guarantee high energy efficiency among UEs with equal number of candidates, ESE gives priority to the UEs with the highest difference in terms of $P_{t o t_{(i, u)}^{t}}$ between their candidates. Subsequently, ESE sorts the candidates of each UE $u$ by $P_{t o t}^{t} t_{(i, u)}$ so that the candidate BS and $\mathrm{BH}$ route combination with the lowest power consumption is favored. In case BS $i$ has sufficient spectrum resources to guarantee the rate of UE $u$ and the selected $\mathrm{BH}$ route does not include BH links that exceed their maximum capacity, UE $u$ gets associated with $\mathrm{BS} i$ and its traffic is routed through $\mathrm{BH}$ path $t$. The algorithm then updates the remaining PRBs of BS $i$ as well as the information regarding the current traffic that passes through each BH link. However, in case the maximum transmission power of at least one $\mathrm{BH}$ link of route $t$ is exceeded, the next BH link route in the list is selected, as long as there is one. Otherwise or if there are no available resources at $\mathrm{BS} i$, the algorithm moves to the next candidate.

Regarding the complexity of the proposed algorithm, it is worth pointing out that the required input information can be easily available to all components of an LTE-A network [4]. In particular, network information can be exchanged among BSs through the X2 interface [4]. Furthermore, information related to the network topology involves very low overhead, as it does not demand frequent updates. Thus, the only extra exchangeable information is the traffic of the BH links, when load balancing is considered. As for the UE reported information, the standard defines the collection of channel state information by the UE for a set of BSs, known as radio resource management (RRM) measurement set. Thereby, the availability of the required information is ensured.

ESE can be applied in each eNB sector as often as needed based on the UE traffic pattern. For quickly changing traffic, a shorter execution time interval would keep the association and $\mathrm{BH}$ routing decision up-to-date, thus leading to better performance, at the expense of higher complexity. Although it is out of the scope of this paper, for best performance, the time interval value should be appropriately selected based on traffic estimation techniques so that the trade-off between complexity and performance is optimized. It is also worth pointing out that for UEs that appear between two consecutive executions of ESE, the UE association and $\mathrm{BH}$ routing could be decided given the associations and $\mathrm{BH}$ routes of the rest of the UEs. Hence, ESE presents high adaptability to changing conditions.

\section{Simulation Results}

To evaluate the performance of the proposed algorithm, we have run extensive simulations in MATLAB ${ }^{\circledR}$. As shown in Fig. 2, we focus on an eNB sector, consisting of two SC clusters of $8 \mathrm{SCs}$ each [11]. According to 3GPP [11], the SC cluster centers are uniformly distributed within the sector, and so are the SCs of each cluster within the cluster region. The SCs should be located at least $20 \mathrm{~m}$ far from each other and $5 \mathrm{~m}$ from the UEs, while the eNB $105 \mathrm{~m}$ from cluster centers and $35 \mathrm{~m}$ from UEs. Furthermore, the three SCs of each cluster that are located the closest to the eNB site play the role of the aggregator of the cluster traffic (i.e., being one hop away from eNB site), the three SCs being the closest to the aggregators are considered two hops away from the eNB site

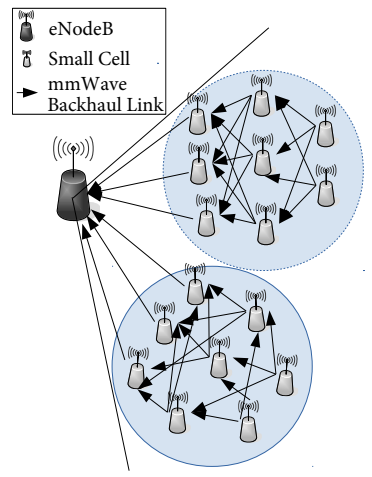

Fig. 2. Simulation scenario.

and the last two SCs of each cluster are considered three hops away. Moreover, the mesh BH network of Fig. 2 is considered.

Regarding the $\mathrm{BH}$, we consider LOS mmWave links operating at $73 \mathrm{GHz}$, while the antenna gains are $43 \mathrm{dBi}$ [3]. Furthermore, to the links that are one-hop-away from the eNB site, we randomly allocate bandwidth from the following set [500, 750, 1000] MHz. Similarly, for the two-hop-away links the set is $[100,200,500] \mathrm{MHz}$, and $[20,50,100] \mathrm{MHz}$ for the three-hop-away links. By virtue of the static nature of $\mathrm{BH}$ links, frequency planning is assumed to be performed initially among neighboring links for interference mitigation.

In each of the 5000 executed iterations, we drop $U$ UEs of different GBR demands. In particular, $70 \%$ of UEs demand $100 \mathrm{Mbps}, 20 \% 150 \mathrm{Mbps}$ and 10\% $300 \mathrm{Mbps}$ [12]. Hotspot traffic is considered, with $2 / 3$ of UEs uniformly distributed within the clusters (in a radius $d=100 \mathrm{~m}$ from cluster center) and $1 / 3$ of UEs uniformly distributed in the eNB sector [11].

Due to constant power allocation, the proposed work is independent of the applied frequency allocation algorithm. Therefore, for simplicity and without loss of generality, we assume that inter-sector interference is mitigated through sophisticated fractional frequency reuse or frequency allocation [15] and that the channels allocated to the eNB are orthogonal to the channels allocated to SCs. Still, SCs of different clusters reuse the same spectrum, thereby interfering to each other.

In Table I, we summarize the rest of the simulation parameters, with $f_{A N}$ denoting the operating frequency of the AN, $B W$ is the allocated bandwidth and $h$ is the antenna height of a BS, SC or UE mobile. The parameter $L_{C B}$ is the cable loss between the radio frequency connector and the antenna of a $\mathrm{BS}$ or $\mathrm{SC}, C_{H}$ is the antenna height correction factor and $d_{i u}$ is the distance between the BS $i$ and UE $u$. Finally, $N F_{U E}$ is the noise figure of a UE. Slow fading is modeled by a log-normal random variable with zero mean and deviation 8 $\mathrm{dB}$ for the eNB and $10 \mathrm{~dB}$ for the SC signal.

The performance of the proposed algorithm is compared with the following low-complexity state-of-the-art solutions ${ }^{3}$.

- ESE: the proposed energy and spectrum efficient algorithm, described in Section III, which considers two different approaches, i.e., with and without load balancing, denoted by ESE with $L B$ and ESE w/o LB, respectively.

- SINR-random: a UE gets associated with the BS with

\footnotetext{
${ }^{3}$ All algorithms are of the same complexity in terms of execution time, $O\left(n^{2} \log n\right)$. Still, they differ in the required information for a UE association.
} 
TABLE I

SiMULATION VALUES

\begin{tabular}{|c|c|c|c|c|c|}
\hline Parameter & Value & Parameter & Value & Parameter & Value \\
\hline$f_{A N}$ & $2.0 \mathrm{GHz}$ & $\bar{c}_{e N B}$ & 100 PRBs & $\overline{N F_{U E} / N F_{B H}}$ & $9 / 6 \mathrm{~dB}$ \\
\hline$B W_{e N B}, B W_{S C}$ & $20 \mathrm{MHz}$ & $G_{T X_{e N B}} / G_{T X_{S C}} / G_{R X_{t}}$ & 19/5/0 dBi & $N_{T H}$ & $-174 \mathrm{dBm} / \mathrm{Hz}$ \\
\hline$C_{H}$ & $0.8+\left(1.1 \log f_{A N^{-}}-0.7\right) h_{U E}-1.56 \log f_{A N}$ & $L_{C B_{c N B}} / L_{C B_{S C}} / L_{T X_{B H}} / L_{R X}$ & $2 / 0 / 5 / 5 \mathrm{~dB}$ & $h_{e N B} / h_{S C} / h_{U E}$ & $25 / 2.5 / 1.5 \mathrm{~m}$ \\
\hline$P L_{e N B}$ & $69.55+26.16 \log f_{A N-1}-13.82 \log h_{e N B^{-}} C_{H}+\left(44.9-6.55 \log h_{e N B}\right) \log \left(d_{i u}\right.$ & $P_{0_{e N B}} / P_{0_{S C}} / P_{0_{B H}}$ & $130 / 6.8 / 3.9 \mathrm{~W}$ & $N_{T R X_{e N B}} / N_{T R X_{S C}} / N_{T R X}$ & $8 / 8 / 1$ \\
\hline$P L_{S C}$ & $69.55+26.16 \log f_{A N}-13.82 \log h_{S C}+\left(44.9-6.55 \log h_{S C}\right) \log \left(d_{i u}\right), d_{i u}$ in $\mathrm{km}$ & $\Delta_{p_{e N B}} / \Delta_{P_{S C}} / \Delta_{p_{B H}}$ & $4.7 / 4 / 4$ & $P_{e N B_{\max }} / P_{S C_{\max }} / P_{B H_{m c}}$ & $46 / 30 / 33 \mathrm{dBm}$ \\
\hline
\end{tabular}

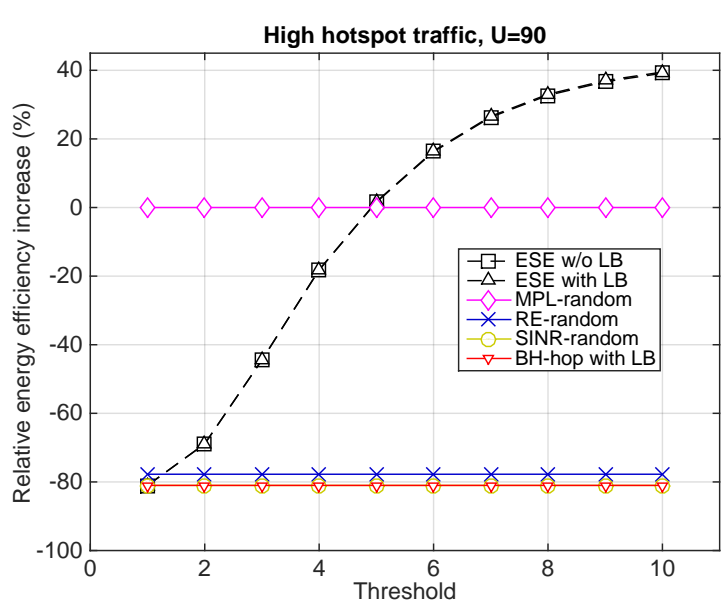

Fig. 3. Relative energy efficiency increase, compared to MPL-random, for high traffic $U=90$ and different threshold values ( $\left.\delta_{\text {thres }}\right)$.

the highest received SINR [4]. As for the BH routing, in the case of a $\mathrm{SC}$ with multiple alternative paths, the algorithm makes a random decision.

- Range expansion (RE)-random: a bias $=13 \mathrm{~dB}$ is added to the SINR if the signal originates from a SC [5], [15]. Regarding the $\mathrm{BH}$ routing, in case of a $\mathrm{SC}$ with multiple alternative paths, the algorithm selects a random path.

- Minimum path loss (MPL)-random: a UE is associated with the BS with the lowest experienced path loss [6], regardless of its received power. As for the $\mathrm{BH}$ routing, in the case of a $\mathrm{SC}$ with multiple alternative paths, the algorithm makes a random decision.

- BH-hop with LB: the energy and spectrum efficient algorithm of [9]. The algorithm i) considers the subset of BSs that maximize the network spectrum efficiency, and from them ii) selects the one with the minimum number of $\mathrm{BH}$ hops to reach the core network. In case of more candidates with equal number of hops, the BS with the less loaded $\mathrm{BH}$ route is selected to achieve load balancing. If there are multiple alternative $\mathrm{BH}$ paths, for a fair comparison, the less loaded $\mathrm{BH}$ route is selected.

To provide a better understanding of the results, in the following graphs, we depict the relative percentage difference in the studied metrics of each algorithm compared to the MPL-random algorithm. Please note that a positive percentage corresponds to an increase in the studied metric compared to MPL-random, whereas a negative to a decrease.

As a result, in Fig. 3 and 4, the relative network energy efficiency and spectrum efficiency increase of all algorithms compared to MPL-random are respectively depicted, under high traffic $(U=90)$, versus the ESE threshold value $\delta_{\text {thres }}$. As it is shown, different $\delta_{\text {thres }}$ only affect the ESE performance, as expected. This is due to the fact that, unlike the rest of the algorithms, ESE approaches are able to provide a variety of trade-offs between the two competitive objectives, i.e., energy and spectrum efficiency, by appropriately selecting the $\delta_{\text {thres }}$

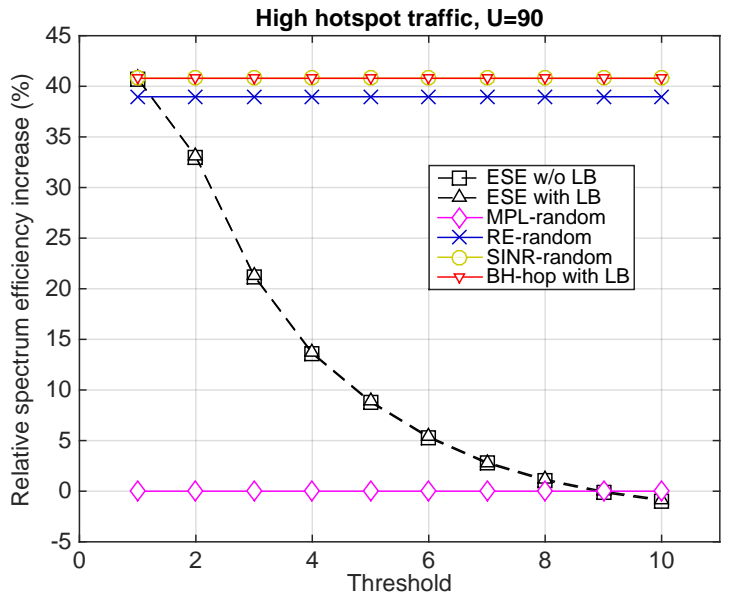

Fig. 4. Relative spectrum efficiency increase, compared to MPL-random, for high traffic $U=90$ and different threshold values $\left(\delta_{\text {thres }}\right)$.

value. A higher threshold corresponds to higher willingness to sacrifice the spectrum efficiency performance in order to increase the energy efficiency. Hence, ESE with $\delta_{\text {thres }}=1$ achieves the maximum spectrum efficiency increase compared to MPL-random at the expense of lower energy efficiency. Nevertheless, for higher $\delta_{\text {thres }}$, the energy efficiency of ESE increases at the expense of lower spectrum efficiency (although still higher than MPL-random for $\delta_{\text {thres }} \leq 9$ ).

Moreover, we can observe that the performance of the proposed ESE approaches is very similar, although they differ in the way they distribute the traffic in the BH links, and thus, in the $\mathrm{BH}$ energy consumption. This is due to the difference in terms of magnitude between the $\mathrm{AN}$ and $\mathrm{BH}$ power consumption, which make the impact of $\mathrm{BH}$ power consumption less apparent in the energy efficiency calculation. Still, ESE with LB slightly outperforms ESE w/o LB, as it distributes the $\mathrm{BH}$ traffic more evenly. Thereby, it leads to low loaded $\mathrm{BH}$ links, and hence, in lower $\mathrm{BH}$ power consumption.

As for the relative difference in energy and spectrum efficiency compared to MPL-random, ESE provides better tradeoffs than MPL-random with $\delta_{\text {thres }}$ values ranging from 5 to 9 , i.e., it provides equal or higher performance in both objectives. For instance, with $\delta_{\text {thres }}=6$, both ESE approaches present almost $20 \%$ improvement in terms of energy efficiency, while, at the same time, they outperform MPL-random in terms of spectrum efficiency by $5 \%$. This is by virtue of the fact that MPL-random associates the UEs independently of their SINR, and thus, it presents very low spectrum efficiency. In parallel, in the considered scenario, where the UEs are located in hotspots, it presents maximum traffic offloading to SCs. This results in very low $\mathrm{AN}$ power consumption (we remind that the power per PRB is much higher for the eNB than for a $\mathrm{SC}$ ) and high $\mathrm{BH}$ power consumption, as there is a lot of $\mathrm{BH}$ traffic that, unlike ESE, is distributed randomly in the links. For the rest of the threshold values, however, no algorithm is better than the other, given that ESE improves one objective 


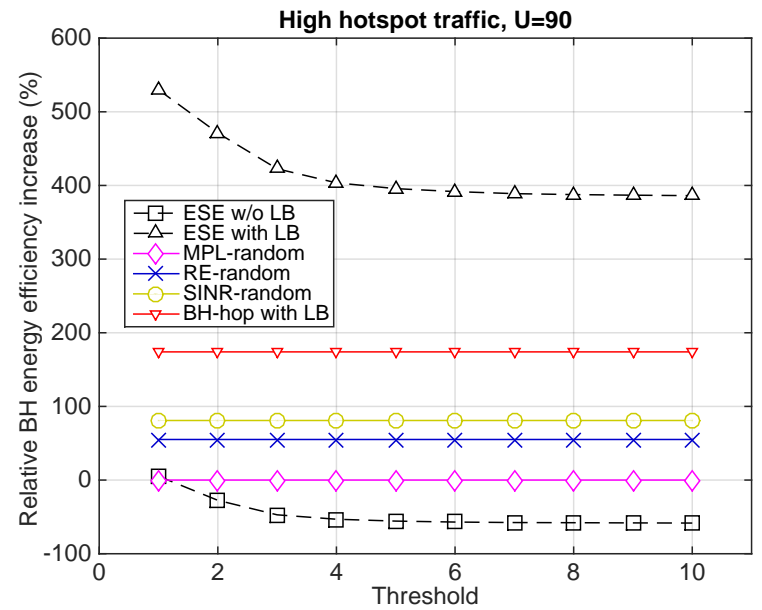

Fig. 5. Relative backhaul energy efficiency increase, compared to MPLrandom, for high traffic $U=90$ and different threshold values $\left(\delta_{\text {thres }}\right)$.

at the expense of the other, thus resulting in another trade-off point. Still, this adaptability on any preference in one of the competitive objectives is an important advantage of ESE.

Regarding the rest of the algorithms, SINR-random and BHhop with LB aim at maximizing the spectrum efficiency, and thus, they achieve more than $40 \%$ spectrum efficiency improvement compared to MPL-random. Still, this comes at the expense of much lower energy efficiency (up to $80 \%$ decrease compared to MPL-random), as more UEs get associated to the eNB, resulting in much higher AN power consumption. On the other hand, RE-random achieves slightly lower spectrum efficiency than the maximum (relative increase $38 \%$ compared to MPL-random) due to the applied bias in favor of slightly higher energy efficiency (78\% lower than MPL-random).

In order to gain a clearer idea on the impact of load balancing, we depict in Fig. 5, the relative BH energy efficiency increase of all algorithms compared to MPL-random for different threshold values. This metric reflects the number of successfully sent bits of traffic that is served through the $\mathrm{BH}$ network divided by the involved $\mathrm{BH}$ energy consumption (measured in bits/Joule). As shown in Fig. 5, ESE with LB and BH-hop with LB achieve the best performance, as expected. Still, the big difference between them is by virtue of the fact that ESE with LB takes into account the possibility of having heterogeneous BH links (i.e., links that differ in length, allocated bandwidth, etc.) and adapts the user association and $\mathrm{BH}$ routing decision accordingly. Thus, by considering the actual power consumption of every BH link and not just the number of hops, it presents lower dependency on the employed scenario, and consequently, lower BH power consumption. Then, for the rest of the algorithms, the higher the number of UEs associated to SCs, and hence, the higher the generated $\mathrm{BH}$ traffic, the lower their performance in terms of $\mathrm{BH}$ energy efficiency. This is due to the fact that the effect of the random $\mathrm{BH}$ route selection in the $\mathrm{BH}$ power consumption becomes more important, as the BH traffic increases. Finally, it is worth pointing out that ESE w/o LB achieves the worst performance, in this case, as it involves high BH traffic and it does not take into account the exponential power growth of the BH links, as traffic increases. Consequently, it results in some links being totally unused, whereas others get very highly loaded, and thus, increase the total $\mathrm{BH}$ power consumption significantly.

\section{CONCLUSION}

The joint user association and $\mathrm{BH}$ traffic routing problem in a $5 \mathrm{G}$ network with mmWave mesh BH links was studied. We proposed a novel algorithm, which bases its user association and $\mathrm{BH}$ routing decision i) on minimizing the required PRBs to guarantee the UE QoS, thus providing high spectrum efficiency, and ii) on minimizing the involved power consumption in both $\mathrm{AN}$ and $\mathrm{BH}$ to provide high energy efficiency. Two different BH routing approaches were considered, i.e, with or without load balancing. The proposed low complexity algorithm was compared with existing solutions and it was shown to be able to provide good trade-offs between the two competitive objectives. Finally, our results demonstrate the high gains of load balancing in lower BH power consumption, and thus, better $\mathrm{BH}$ resource utilization. As future work, we will compare the proposed approaches with optimal solutions obtained through analytical methods. Despite their high complexity, the optimal solutions will be valuable to further prove the gains of the proposed low-complexity approaches.

\section{ACKNOWLEDGMENT}

Part of this work has been funded by the Knowledge Foundation (KK-Stiftelsen) of Sweden through the Project SOCRA and by the Spanish Government and ERDF through CICYT project TEC2013-48099-C2-1-P.

\section{REFERENCES}

[1] Cisco visual networking index: forecast and methodology, 2015-2020, white paper, Jun. 2016.

[2] "NGMN 5G white paper," NGMN Alliance, v. 1.0, Feb. 2015.

[3] A. Mesodiakaki et al., "Energy efficient line-of-sight millimeter wave small cell backhaul: 60, 70, 80 or $140 \mathrm{GHz}$ ?," in Proc. IEEE WoWMoM, Jun. 2016, pp. 1-9.

[4] E-UTRA and E-UTRAN; Overall description; Stage 2, 3GPP TS 36.300, v. 11.5.0, Rel. 11, Mar. 2013.

[5] "DL pico/macro HetNet performance: cell selection," Alcatel-Lucent, R1100945, Meeting 60, Feb. 2010.

[6] D. Fooladivanda and C. Rosenberg, "Joint resource allocation and user association for heterogeneous wireless cellular networks," IEEE Trans. Wireless Commun., vol. 12, no. 1, pp. 248-257, Oct. 2012.

[7] N. Sapountzis, T. Spyropoulos, N. Nikaein, and U. Salim, "User association in over- and under- provisioned backhaul HetNets," Eurocom Research Report, 16-318, Apr. 2016.

[8] Q. Han, B. Yang, C. Chen, and X. Guan, "Backhaul-aware joint user association and resource allocation for energy-constrained HetNets," IEEE Trans. Veh. Technol., vol. 66, no. 1, pp. 580-593, Jan. 2017.

[9] A. Mesodiakaki, F. Adelantado, L. Alonso, and C. Verikoukis, "Energyefficient context-aware user association for outdoor small cell heterogeneous networks," in Proc. IEEE ICC, Jun. 2014, pp. 1614-1619.

[10] A. Mesodiakaki et al., "Energy and spectrum efficient user association in millimeter wave backhaul small cell networks," IEEE Trans. Veh. Technol, vol. 66, no. 2, pp. 1810-1821, Feb. 2017.

[11] Small cell enhancements for E-UTRA \& E-UTRAN-Physical layer aspects, 3GPP TR 36.872, v. 1.0.0, Rel. 12, Aug. 2013.

[12] Study on small cell enhancements for E-UTRA and E-UTRAN; Higher layer aspects, 3GPP TR 36.842, v. 12.0.0, Rel. 12, Dec. 2013.

[13] G. Auer et al., "How much energy is needed to run a wireless network?," IEEE Wireless Commun., vol. 18, no. 5, pp. 40-49, Oct. 2011.

[14] E. Dahlman, S. Parkvall and J. Skld, 4G: LTE/LTE-Advanced for Mobile Broadband, Academic Press, ISBN: 012385489X, 2011.

[15] D. Lopez-Perez, X. Chu, and I. Guvenc, "On the expanded region of picocells in heterogeneous networks," IEEE J. Sel. Topics Signal Process., vol. 6, no. 3, pp. 281-294, Jun. 2012. 